\title{
Placas columnares de crinoideos de la Formación Las Delicias, Pérmico inferior y medio de Coahuila, México
}

\author{
Columnar crinoid plates from Las Delicias Formation, lower and middle Permian of Coahuila, \\ Mexico
}

Rafael Villanueva-Olea ${ }^{1}$, Sara Alicia Quiroz-Barroso ${ }^{1}$, Jesús Quiroz-Barragán ${ }^{2}$, Miguel Ángel Torres-Martínez ${ }^{3}$, Francisco Sour-Tovar ${ }^{1, *}$

\begin{abstract}
Departamento de Biología Evolutiva, Facultad de Ciencias, Universidad Nacional Autónoma de México. Ciudad Universitaria, 04510, Coyoacán, CDMX, México.

${ }^{2}$ Museo Paleontológico de la Laguna. Av. Juárez 580 Pte. Centro, 27000, Torreón, Coahuila, México.
\end{abstract}

${ }^{3}$ Departamento de Paleontología, Instituto de Geología, Universidad Nacional Autónoma de México. Ciudad Universitaria, 04510, Coyoacán, CDMX, México.

* Autor para correspondencia: (F. Sour-Tovar) fcosour@ciencias.unam.mx

\section{RESUMEN}

Se describen trece parataxones de placas columnares de crinoideos que se han encontrado en dos localidades del Pérmico inferior y medio de la Formación Las Delicias. Ambas se ubican en la Sierra de las Delicias, al suroeste del estado de Coahuila. En estratos del Pérmico medio (Wordiano-Capitaniano) de la localidad Las Difuntas se encontró a Pentagonopternix coahuilensis $\mathrm{n}$. sp., Cyclocaudex typicus, $C$. insaturatus, C. cf. insaturatus, Cyclocaudex sp., Floricyclus diminuta n. sp., Floricyclus sp., Preptopremnum rugosum, P. laeve, Preptopremnum sp., Heterostelechus keithi, Cyclocaudiculus regularis y Epicrinus torreonense gen. et sp. nov. En estratos de la localidad Las Sardinas, cuya edad es Pérmico temprano (Kunguriano-Roadiano), también se presenta Cyclocaudex typicus, C. sp., Preptopremnum laeve y a Epicrinus torreonense. Para todos los parataxones presentes en la Formación Las Delicias, que han sido referidos previamente para otras localidades, se establece un alcance estratigráfico que se amplía hasta el Wordiano-Capitaniano. Los parataxones descritos se asocian, en su mayoría, a la Paleoprovincia Grandiana y la presencia de especies de Floricyclus y Pentagonopternix en el Pérmico de Coahuila comprueba el carácter cosmopolita de ambos géneros.

Palabras clave: Crinoidea, placas columnares, Pérmico, Formación Las Delicias, Coahuila, México.

\section{ABSTRACT}

Thirteen parataxa of crinoid plates from two localities of the lower-middle Permian of the Las Delicias Formation are described. Both zones are situated in Sierra de las Delicias, to southwestern Coahuila state. The parataxa Pentagonopternix coahuilensis n. sp., Cyclocaudex typicus, C. insaturatus, C. cf. insaturatus, Cyclocaudex sp., Floricyclus diminuta $n$. sp., Floricyclus sp. Preptopremnum rugosum, $P$. laeve, Preptopremnum sp., Heterostelechus keithi. Cyclocaudiculus regularis and Epicrinus torreonense new genus and species were found in Las Difuntas locality of the middle Permian (Wordian-Capitanian) whereas Cyclocaudex typicus, C. sp., Preptopremnum laeve and Epicrinus torreonense were also found in Las Sardinas locality whose strata have been dated as early Permian (Kungurian-Roadian). The occurrence of all parataxa in strata from the middle Permian of the Las Delicias Formation allowed extending their stratigraphic range until the Wordian-Capitanian. Most species described have been associated to the Grandian Paleoprovince, nonetheless, the presence of Floricyclus and Pentagonopternix parataxa in the Permian of Coahuila proves the cosmopolitan nature of both genera.

Keyzords: Crinoidea, columnal plates, Permian, Las Delicias Formation, Coahuila, México.
La revisión por pares es responsabilidad de la Universidad Nacional Autónoma de México.

Este es un artículo Open Access bajo la licencia CC Y-NCSA (https://creativecommons.org/licenses/by-nc-sa/4.0/) 


\section{Introducción}

El registro fósil de crinoideos es común y abundante en rocas de todo el Fanerozoico, pero se caracteriza, principalmente, por la conservación diferencial y desarticulada de los componentes que conforman el esqueleto de cada individuo y por la poca existencia de casos en que se encuentren articuladas todas las partes. Este sesgo es producto de la forma en que se articulan el pedúnculo, cáliz, brazos y cirros que conforman al individuo, partes que, a su vez, están compuestas por una serie de placas u osículos de calcita y magnesio que se unen entre sí por suturas rígidas o flexibles. Al morir el organismo, dichas estructuras no son capaces de mantener su función, ocurriendo rápidamente la desarticulación post-mortem de los crinoideos. Por ello lo común es encontrar placas desarticuladas que difícilmente permiten reconocer al taxón al que pertenecieron. Considerando este sesgo, diversos investigadores que han trabajado con registros paleozoicos, en particular Moore y Jeffords (1968), han propuesto un sistema de clasificación basado en la morfología de las placas y han tratado de desarrollar un esquema sobre la distribución temporal y espacial de cada morfotipo con la idea de que el registro de este tipo de fósiles sea de utilidad bioestratigráfica o paleobiogeográfica.

En este contexto, cada trabajo en que se describen partes sueltas de crinoideos representa una aportación en la elaboración de tal esquema. Siguiendo la idea, y dado que no existen trabajos previos en que se mencione o describa el registro fósil de crinoideos del Pérmico de la Sierra de las Delicias, este trabajo tiene como objetivo presentar las descripciones y las determinaciones de una serie de placas columnares que se han encontrado en diversos niveles estratigráficos del Pérmico inferior-medio de la Formación Las Delicias. Con ello se espera aportar y ampliar la información existente sobre la distribución espacio-temporal de placas columnares aisladas de crinoideos. Un segundo objetivo es discutir las implicaciones bioestratigráficas y paleogeográficas de la presencia del material estudiado.

\section{Marco Geológico}

La Formación Las Delicias se caracteriza por una dominancia de estratos de lutita y caliza, escombros volcánicos dacíticos y andesíticos, capas de sedimentos marinos finos de origen pelágico y estructuras sedimentarias propias de eventos turbidíticos (McKee et al., 1999). Los mejores afloramientos de esta unidad se encuentran expuestos en las sierras Las Delicias, Las Margaritas y Los Remedios, y, en menor medida, en la Sierra Tlahualillo y en el Valle de San Marcos, todas unidades geomorfológicas ubicadas al noroeste de la ciudad de Torreón, en el extremo suroeste del estado de Coahuila en el norte de México (Figura 1, parte superior).

Los afloramientos de la Formación Las Delicias cubren un área de $6000 \mathrm{~km}^{2}$ aproximadamente y contienen a las rocas pérmicas más jóvenes de Norteamérica (McKee et al., 1999). Constituyen los depósitos de la Cuenca de Las Delicias, para la cual se ha propuesto que se encontraba adyacente a un arco magmático que posiblemente se formó en la parte noroeste de Gondwana (McKee et al., 1990; Sedlock et al., 1993) y actualmente forma parte del Bloque de Coahuila o Terreno Coahuiltecano (Coney y Campa-Uranga, 1987).

\section{Estratigrafía}

Dada la poca continuidad lateral de las rocas paleozoicas en la cuenca de Las Delicias y al no encontrarse aún contactos claros entre las diferentes subunidades, las correlaciones estratigráficas entre las rocas del Paleozoico Superior de la región son dificiles de establecer. Además, movimientos post deposicionales provocaron que la gran mayoría de las capas de sedimentos pérmicos fueran cubiertas por coladas de rocas mesozoicas. Dados estos problemas, Wardlaw et al. (1979) dividieron la sucesión de rocas pérmicas de la Sierra de las Delicias en cinco unidades informales: 1) la basal, llamada las Sardinas, que fue asignada al Kunguriano-Roadiano (Cisuraliano tardío- 


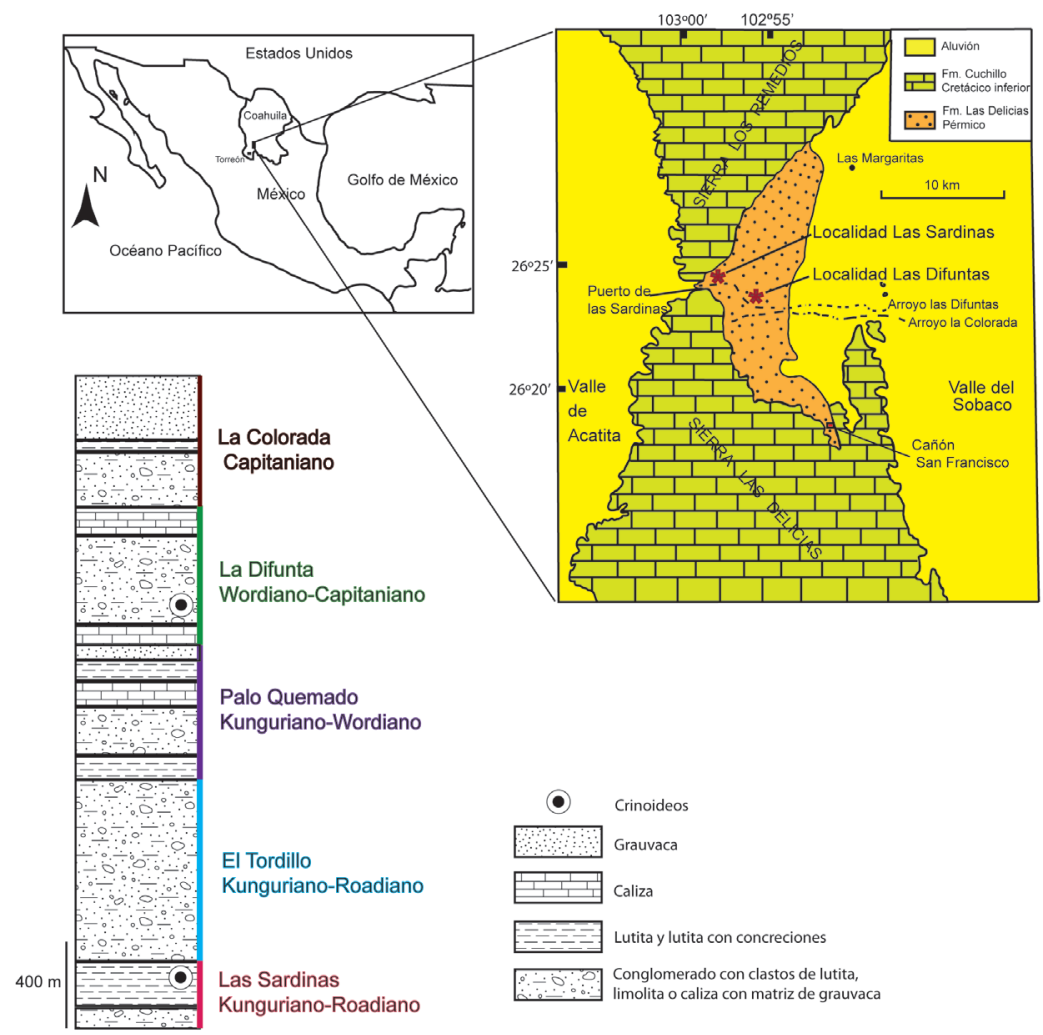

Figura 1 Parte superior, mapas que señalan la ubicación de las localidades Las Sardinas y Las Difuntas. Parte Inferior, sección esquemática de las unidades informales y sus edades, propuestas por Wardlaw et al. (1979) para el Pérmico de la Sierra de Las Delicias. En esta sección se señalan los niveles estratigráficos en que se recolectaron las placas columnares de crinoideos que se describen en este trabajo. (Modificado de Quiroz-Barroso et al., 2019).

Guadalupiano temprano), seguida por 2) el Tordillo del Kunguriano-Rodiano (Cisuraliano tardío-Guadalupiano temprano), 3) Palo Quemado del Kunguriano-Wordiano (Guadalupiano medio), 4) la Difunta del Wordiano-Capitaniano (Guadalupiano medio-tardío) y 5) la Colorada del Capitaniano (Guadalupiano tardío).

Por otro lado, McKee et al. (1999), al describir las características de la Formación Las Delicias, propusieron no utilizar el término "Miembros" por las implicaciones litoestratigráficas que conlleva, y, en cambio, emplear el término informal de "Litosoma". De acuerdo con dichos autores, existen dos tipos de litosomas en el área, unos "Regulares o Estratificados" que se depositaron durante regímenes de sedimentación típicos. El segundo tipo agrupa litosomas que se formaron por el desplazamiento caótico de grandes cuerpos de roca, con volúmenes de pocos hasta cientos de $\mathrm{m}^{3}$. Para la región de las Delicias se describieron 11 subunidades en total: 4 litosomas estratificados (Los Remedios, Los Angelitos, Las Parritas y La Cría) y 7 litosomas caóticos (El Fraile, Los Piloncillos, La Venada, El Fierro, Cañón Rosillo, Cerro Prieto y San Pedro).

Los ejemplares que se describen en este trabajo se recolectaron en dos localidades:

1. Afloramientos ubicados en los alrededores de Puerto Sardinas, con estratos de la base de la Formación Las Delicias, correspondientes a la unidad que Wardlaw et al. (1979) nominaron Las Sardinas y que King et al. (1944) describieron como la Lutita Perrinites marcando una edad del Kunguriano-Roadiano (Cisuraliano tardíoGuadalupiano temprano). En la base de esta sección se encuentran rocas conglomeráticas seguidas por areniscas y lutitas interestratificadas. 
2. En estratos de la sección tipo de la Formación Las Delicias, en el arroyo de Las Difuntas, y que son correspondientes a la unidad La Difunta propuesta por Wardlaw et al. (1979). En dicha unidad son abundantes, entre otros invertebrados fósiles, Waagenoceras dieneri, $W$. karpinsky, Pseudagathiceras difuntense, Eumedlicottia burckhardti y otros cefalópodos que marcan una edad del Wordiano-Capitaniano (Guadalupiano medio). La sección se caracteriza por lutita en su base, seguida por intercalaciones de calcarenita, estratos delgados de caliza y arenisca. En la parte superior de esta sección se encuentra un banco de caliza de origen arrecifal rica en esponjas, braquiópodos richtofénidos y fusulínidos.

Los dos afloramientos portadores de placas de crinoideos forman parte del litosoma de La Cría que aflora principalmente en las sierras de Las Delicias y Malascachas, presenta un espesor de cerca de $2500 \mathrm{~m}$ con estratos delgados de arenisca volcánica, conglomerado y turbiditas que llegan a presentar marcas de flujo de tipo contourita y otras evidencias de deslizamientos. Ocasionalmente se encuentran cantos de caliza de gran tamaño. Este litosoma propuesto por McKee et al. (1999) corresponde con las cinco subunidades que plantearon Wardlaw et al. (1979) para la Formación Las Delicias y, a su vez, con la secuencia pérmica propuesta por King et al. (1944). En la parte inferior de la Figura 1 se ilustra la sección pérmica de la Sierra de las Delicias y se nombran las cinco unidades que conforman la Formación Las Delicias y las edades que se le han asignado (Wardlaw et al., 1979).

\section{Paleoambiente}

Considerando las ideas de McKee et al. (1999), la Formación Las Delicias representa una serie de depósitos que se acumularon en un ambiente asociado a un arco volcánico. Las características sedimentológicas indican aguas tranquilas con flujos estacionales de terrígenos e influencia de eventos volcánicos. En este contexto, la acumulación en los estratos kungurianoscapitanianos de la Formación Las Delicias de una gran diversidad y abundancia de invertebrados, tanto bentónicos como nectónicos, incluyendo los crinoideos que se analizan en este trabajo, se puede explicar considerando eventos de flujo de sedimentos de zonas someras hacia zonas semiprofundas. Un caso similar se ha descrito para el Pérmico del estado de Hidalgo (Rosales et al., 2005), en donde se encontraron asociaciones de invertebrados bentónicos de aguas someras en flujos de volcaniclastos y terrígenos finos que se depositaron en ambientes marinos profundos.

\section{Paleontología sistemática}

Para la determinación taxonómica de los ejemplares se siguió el sistema de clasificación parataxonómica de Moore yJeffords (1968), basado en el estudio de placas columnares y columnas de crinoideos paleozoicos. Los ejemplares con las siglas MPLL se encuentran depositados en el Museo Paleontológico de la Laguna, en Torreón, Coahuila, mientras que los que presentan las siglas FCMP forman parte de la colección de Ejemplares Tipo del Museo de Paleontología de la Facultad de Ciencias de la Universidad Nacional Autónoma de México.

$$
\begin{gathered}
\text { Subclase y Orden Inciertos } \\
\text { [Grupo Pentameri] }
\end{gathered}
$$

Familia Pentacauliscidae Moore y Jeffords, 1968

Género Pentagonopternix Moore y Jeffords, 1968

Pentagonopternix coahuilensis n. sp.

Figuras 2.1, 2.2.

Diagnosis. Placas de forma pentaestelada de alturas diferentes; suturas de indentadas a crenuladas; faceta articular con un cinturón de crénulas gruesas y de corto tamaño; crenulario con disposición floriforme ubicado hacia el interior de la placa, cerca, sin hacer contacto, del margen formando rayos petaloides; limita con una areola 

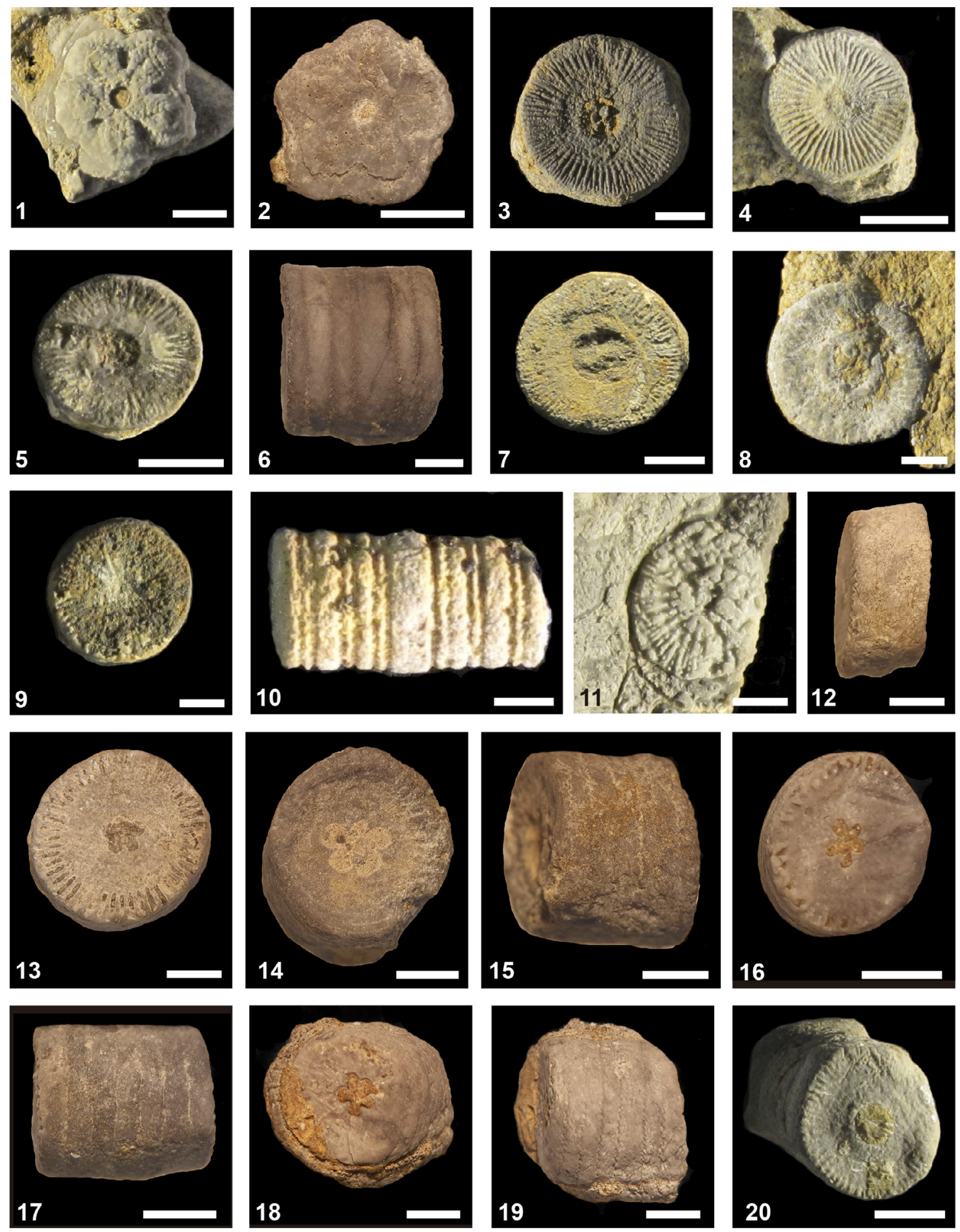

Figura 2 (1, 2) Pentagonopternix coahuilensis n. sp., 1, faceta articular, escala $3 \mathrm{~mm}$, ejemplar MPLL 17; 2, faceta articular, escala 3 mm, ejemplar MPLL 18. (3-6) Cyclocaudex typicus, 3, faceta articular, escala 6 mm, ejemplar MPLL 19; 4, faceta articular, escala 6 mm, ejemplar FCMP 1334; 5, faceta articular, escala 6 mm, ejemplar MPLL 20; 6, Perfil longitudinal de la columna, escala 3 mm, ejemplar MPLL 20. (7-8) Cyclocaudex insaturatus, 7, faceta articular, escala 6 mm, ejemplar FCMP 1340; 8, faceta articular, escala 3 mm, ejemplar FCMP 1341 . (910) Cyclocaudex cf. insaturatus, 9, faceta articular, escala $3 \mathrm{~mm}$, ejemplar FCMP 1338; 10, perfil longitudinal de la columna, escala $3 \mathrm{~mm}$, ejemplar FCMP 1339. (11) Cyclocaudex sp., faceta articular, escala $3 \mathrm{~mm}$, ejemplar FCMP 1333. (12, 13) Floricyclus diminuta, n. sp., 12, perfil longitudinal de la columna, escala $3 \mathrm{~mm}$, ejemplar MPLL 29; 13, faceta articular, escala 3 mm, ejemplar MPLL 29. (14-19) Floricyclus sp., 14, faceta articular, escala $3 \mathrm{~mm}$, ejemplar MPLL 24; 15, Perfil longitudinal de la columna. Escala $3 \mathrm{~mm}$. No. de catálogo MPLL 24; 16, faceta articular, escala $3 \mathrm{~mm}$, ejemplar MPLL 25; 17, perfil longitudinal de la columna, escala $3 \mathrm{~mm}$, ejemplar MPLL 25; 18, Faceta articular, escala $3 \mathrm{~mm}$, ejemplar MPLL 26; 19, perfil longitudinal de la columna, escala $3 \mathrm{~mm}$, ejemplar MPLL 26. (20) Preptopremnum rugosum, faceta articular, escala $6 \mathrm{~mm}$, ejemplar MPLL 30. 
pentaestelada lisa, que forma el interior de cada uno de los rayos petaloides de la faceta y rodea al lumen, el cual es pequeño, de forma circular y con un yúgulo pentagonal.

Discusión. El ejemplar de Coahuila fue comparado con Pentagonopternix insculptus del Pensilvánico Superior de Texas (Moore y Jeffords, 1968) que presenta un crenulario con contorno pentaestelado y areola cóncava. Por el contrario, en la especie de Coahuila el margen del crenulario es floriforme y la areola es plana.

Ocurrencia. Formación Las Delicias, Coahuila, México, base de la Unidad La Difunta, Pérmico medio (Wordiano-Capitaniano).

Distribución. El género Pentagonopternix se ha encontrado en el Pensilvánico Medio de Sierra Agua Verde, Sonora, México (Buitrón-Sánchez et al., 2015) y en la Caliza Chaffin, Grupo Cisco, del Virgiliano de Texas, Estados Unidos (Moore y Jeffords, 1968). En el Pérmico se reporta para la Formación Grupera del Asseliano-Sakmariano de Chiapas, México (Torres-Martínez et al., 2020), para el Horizonte Antiinskiy del Pérmico medio (Roadiano-Wordiano) en la región Transbaikal de Rusia (Kotlyar et al., 2006) y para la Formación Imtachan del Pérmico superior de Verkhoyansk, Rusia (Biakov et al., 2016).

Ejemplares tipo. Holotipo: MPLL 17. Paratipo: MPLL 18.

[Grupo Cyclici]

Familia Cyclomischidae Moore y Jeffords, 1968 Género Cyclocaudex Moore y Jeffords, 1968 Cyclocaudex typicus Moore y Jeffords, 1968 Figuras 2.3-2.6.

Descripción. Placa de contorno circular con lados rectos y lisos; crenulario no contacta al lumen y es de tamaño medio con crénulas delgadas, la mayoría bifurcadas; areola estrecha y ligeramente deprimida rodeando al lumen, el cual es pequeño y circular.

Discusión. El material de Cyclocaudex typicus de Coahuila se caracteriza por la areola que rodea al lumen y se diferencia de Cyclocaudex insaturatus, cuyo crenulario está en contacto directo con el lumen. Cyclocaudiculus regularis se distingue por crénulas más gruesas y en menor número que las presentes en C. typicus.

Ocurrencia. Formación Las Delicias, Coahuila, México, Unidad Las Sardinas, Pérmico inferior (Kunguriano) y base de la Unidad La Difunta, Pérmico medio (Wordiano-Capitaniano).

Distribución. Cyclocaudex typicus se ha encontrado en la formación Santiago del MeramecianoChesteriano (Misisípico) de Santiago Ixtaltepec, Oaxaca (Villanueva-Olea et al., 2011). Para el Pensilvánico, se reporta en la Formación Ixtaltepec del Morrowano-Desmoinesiano de Santiago Ixtaltepec, Oaxaca (Villanueva-Olea et al., 2011); en la Formación Patlanoaya del Pensilvánico Superior de Puebla, México (Villaseñor-Martínez et al., 1987; Esquivel-Macías, 1996) y en Estados Unidos, en la Formación Thrifty, Grupo Cisco del Virgiliano de Texas (Moore y Jeffords, 1968). Para el Pérmico se tiene registro en la Formación La Cruz del Wordiano temprano-medio de Ihualtepec, Oaxaca, México (Vachard et al., 1997), y en la Formación Grupera del AsselianoSakmariano de Chiapas, México (Torres-Martínez et al., 2020).

Material descrito. FCMP 1334, FCMP 1335, FGMP 1336, FGMP 1337, MPLL 19, MPLL 20, MPLL 21.

\section{Cyclocaudex insaturatus Moore y Jeffords, 1968}

Figuras 2.7, 2.8.

Descripción. Placa de contorno circular con lados rectos y lisos; suturas crenuladas; faceta articular con un crenulario muy ancho, con crénulas muy delgadas y rectas que llegan hasta el borde del lumen; cúlmenes más delgados que las crenelas; areola ausente; lumen grande y circular. Discusión. Los ejemplares de Coahuila son similares a Avicantus dunbari, del Devónico Inferior de Tennessee (Moore y Jeffords, 1968), en la falta de areola y por la gran anchura del crenulario, no 
obstante, las placas de Avicantus dunbari tienen un perfil longitudinal muy convexo y presencia de costillas. Otras especies de Cyclocaudex se pueden diferenciar de Cyclocaudex insaturatus por presentar una areola o un perilumen conspicuo.

Ocurrencia. Formación Las Delicias, Coahuila, México, base de la Unidad La Difunta, Pérmico medio (Wordiano-Capitaniano).

Distribución. Cyclocaudex insaturatus se encontró en la Formación Santiago del Misisípico Medio de Santiago Ixtaltepec, Oaxaca, México (VillanuevaOlea et al., 2011). Para el Pensilvánico hay reportes sobre su presencia en la Formación Del Monte del Pensilvánico de Calnali, Hidalgo, México (Buitrón-Sánchez et al., 1987), en la Formación Ixtaltepec del Pensilvánico Inferior-Medio de Santiago Ixtaltepec, Oaxaca, México (BuitrónSánchez et al., 2000) y en la Formación Canabiss del Desmoinesiano de Kansas, Estados Unidos. (Moore y Jeffords, 1968). Para el Pérmico se ha registrado en la Formación Grupera del AsselianoSakmariano de Chiapas, México (Torres-Martínez et al., 2020).

Material descrito. FCMP 1340, FCMP 1341, FGMP 1342, FGMP 1343.

\section{Cyclocaudex cf. insaturatus Moore y Jeffords, 1968}

Figuras 2.9, 2.10.

Descripción. Columna heteromórfica, con placas de baja altura en al menos 4 niveles diferentes (internodales 3IN); lados de las placas redondeados; la faceta articular ocupa el diámetro total de la placa; crenulario ancho conformado de crénulas muy delgadas y rectas; areola y crenulario no preservados.

Discusión. El material de la Formación Las Delicias presenta depósitos de sedimento que enmascaran varios rasgos de la areola y el lumen y que impiden una determinación más confiable. Es similar a Cyclocaudex insaturatus del Pensilvánico Medio de Kansas, Estados Unidos (Moore y Jeffords, 1968), por ofrecer crénulas muy delgadas y largas que parecen llegar hasta el centro de la faceta en el límite del lumen. El ejemplar de Kansas muestra una diferencia no tan marcada en la altura de las placas, la cual sí es notable en el ejemplar de Coahuila.

Ocurrencia. Formación Las Delicias, Coahuila, México, base de la Unidad La Difunta, Pérmico medio (Wordiano-Capitaniano).

Material descrito. FGMP 1338, FGMP 1339.

\section{Cyclocaudex sp.}

Figura 2.11.

Descripción. Columna heteromórfica, con presencia de internodales hasta de tercer orden (3IN); suturas crenuladas; placa de contorno circular; crenulario ancho, con crénulas finas, la mayoría con bifurcaciones a la altura media del crenulario; areola no visible; lumen pequeño y circular.

Discusión. Los detalles de la faceta articular del ejemplar de Coahuila lo asocian al género Cyclocaudex. El tipo de columna heteromórfica, con internodales de hasta tercer orden, lo caracterizan como una posible nueva especie del género, sin embargo, no se cuenta con ejemplares mejor preservados que permitan aseverar la propuesta. El ejemplar fue comparado con Cylindrocauliscus fiski, del Pensilvánico Medio de Texas, Estados Unidos (Moore y Jeffords, 1968), que se distingue por sus crénulas más gruesas y un crenulario con más anchura.

Ocurrencia. Formación Las Delicias, Coahuila, México, base de la Unidad La Difunta, Pérmico medio (Wordiano-Capitaniano).

\section{Material descrito. FGMP 1333.}

Familia Floricyclidae Moore y Jeffords, 1968 Género Floricyclus Moore y Jeffords, 1968 Floricyclus diminuta $\mathrm{n}$. sp.

Figuras 2.12, 2.13.

Diagnosis. Columna heteromórfica con perfil longitudinal recto y liso en los internodales 
conservados y con perfil convexo para la placa nodal, lo que le proporciona un diámetro ligeramente mayor a esta última; placa de contorno circular; faceta articular que ocupa todo el diámetro de la placa; crenulario de tamaño medio con crénulas rectas y de grosor medio; areola ancha y lisa; lumen floriforme de tamaño pequeño.

Discusión. El ejemplar fue comparado con las diferentes especies del género Floricyclus (Moore y Jeffords, 1968) que se distinguen por un lumen floriforme más grande, presentar una areola más pequeña y granulaciones alrededor del lumen.

Derivación del nombre. El nombre de la especie "diminuta" hace referencia al carácter pequeño del lumen floriforme.

Ocurrencia. Formación Las Delicias, Coahuila, México, base de la Unidad La Difunta, Pérmico medio (Wordiano-Capitaniano).

Holotipo. MPLL 29.

\section{Floricyclus sp.}

Figuras 2.14-2.19.

Descripción. Placa de contorno circular; faceta articular que ocupa la mayor parte del diámetro de la placa o la ocupa por completo; crenulario aparentemente estrecho; areola ancha, en general lisa o levemente deprimida; lumen floriforme de tamaño medio.

Discusión. Los ejemplares de Coahuila se asignan al género Floricyclus (Moore y Jeffords, 1968) por la presencia del lumen petaloide. Se compararon con el género Cyclocion, del Misisípico Superior de Illinois (Moore y Jeffords, 1968), que se distingue por un lumen de mayor tamaño.

Ocurrencia. Formación Las Delicias, Coahuila, México, base de la Unidad La Difunta, Pérmico medio (Wordiano-Capitaniano).

Distribución. El género Floricyclus se ha reportado en estratos del Misisípico, específicamente en Pilton Beds, Fremington Quay del Tournaisiano de Devon, Inglaterra (Gluchowski y Racki, 2005), en la Formación Brodhead, Grupo Borden del Osageano de Kentucky (Moore y Jeffords, 1968) y en la Formación Santiago del Misisípico Medio de Santiago Ixtaltepec, Oaxaca, México (VillanuevaOlea et al., 2011). Durante el Pensilvánico, se reporta para la Lutita Mingus, Grupo Strawn del Desmoinesiano de Texas, Estados Unidos, (Moore y Jeffords, 1968), la Formación Minturn del Desmoinesiano de Colorado, Estados Unidos (Moore y Jeffords, 1968), la Formación Canabiss, Grupo Cherokee del Desmoinesiano de Kansas, Estados Unidos (Moore y Jeffords, 1968), la Formación Thrifty, Grupo Cisco del Virgiliano de Texas, Estados Unidos (Moore y Jeffords, 1968) y la Formación Patlanoaya del Pensilvánico MedioSuperior de San Salvador Patlanoaya, Puebla, México (Esquivel-Macías et al., 2000).

Material descrito. MPLL 24, MPLL 25, MPLL 26, MPLL 27, MPLL 28.

Familia Leptocarphiidae Moore y Jeffords, 1968

Género Preptopremnum Moore y Jeffords, 1968

Preptopremnum rugosum Moore y Jeffords, 1968

Figuras 2.20, 3.1, 3.2.

Descripción. Columna heteromórfica con una noditaxis de nueve columnales; presencia de nodales con dos a tres cicatrices cirrales, más anchos y más altos que los internodales; internodales de primero, segundo y tercer orden; perfil longitudinal de la columna recto a redondeado; placa de contorno circular; crénulas gruesas y rectas; areola de tamaño medio, deprimida y ligeramente granular en un ejemplar debido a la conservación; perilumen elevado rodeando un crenulario circular, de tamaño medio, que presenta un declive hacia el interior de la placa.

Discusión. Los ejemplares de Coahuila fueron comparados con Heterostelechus jeffordsi y H. keithi, ambos del Pensilvánico Superior de Texas, Estados Unidos (Moore y Jeffords, 1968). Las dos especies poseen crénulas mucho más gruesas y el perilumen no está elevado. Cyclocaudiculus regularis del Pensilvánico Superior de Texas, Estados Unidos (Moore y Jeffords, 1968), es similar al espécimen de Coahuila, pero en el primero no es evidente un perilumen. 

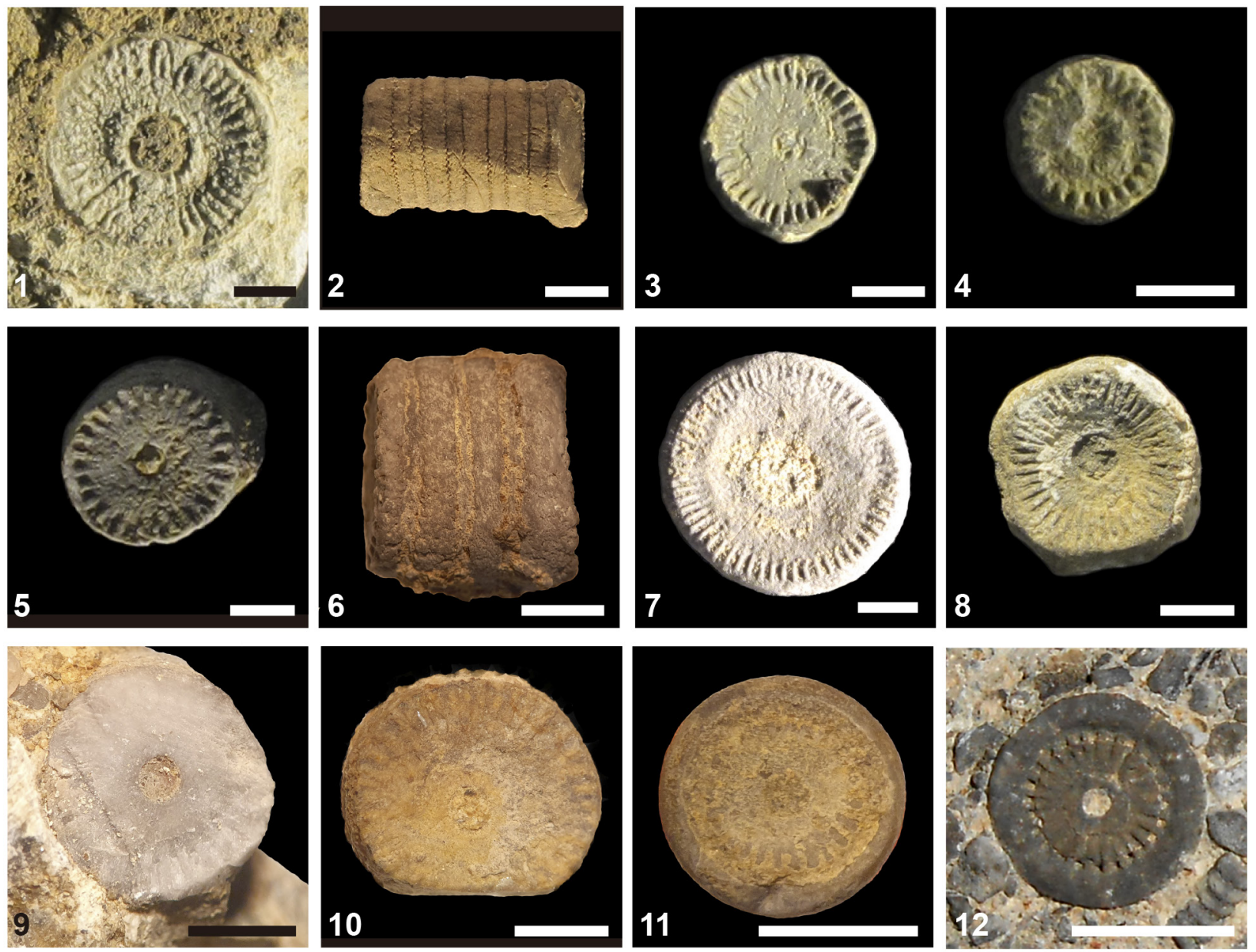

Figura 3 (1-2) Preptopremnum rugosum, 1, faceta articular, escala 3 mm, ejemplar FCMP 1345; 2, perfil longitudinal de la columna, escala 6 mm, ejemplar MPLL 30. (3-6) Preptopremnum laeve, 3, faceta articular, escala $3 \mathrm{~mm}$, ejemplar MPLL 32; 4, faceta articular, escala $3 \mathrm{~mm}$, ejemplar MPLL 33; 5, faceta articular, escala $3 \mathrm{~mm}$, ejemplar MPLL 34; 6, perfil longitudinal de la columna, escala $3 \mathrm{~mm}$, ejemplar MPLL 34; (7) Preptopremnum sp., faceta articular, escala $3 \mathrm{~mm}$, ejemplar FCMP 1344; (8) Heterostelechus keithi, faceta articular, escala $6 \mathrm{~mm}$, ejemplar MPLL 35. (9,10) Cyclocaudiculus regularis, 9, faceta articular, escala $3 \mathrm{~mm}$, ejemplar FCMP 1347; 10, faceta articular, escala $3 \mathrm{~mm}$, ejemplar FCMP 1348. (11, 12) Epicrinus torreonense gen. et sp. nov., 11, faceta articular, escala $3 \mathrm{~mm}$, ejemplar MPLL 37; 12, faceta articular, escala $3 \mathrm{~mm}$, ejemplar MPLL 38.

Ocurrencia. Formación Las Delicias, Coahuila, México, base de la Unidad La Difunta, Pérmico medio (Wordiano-Capitaniano).

Distribución. Preptopremnum rugosum se ha encontrado en el Misisípico de San Salvador Patlanoaya, Puebla, México (Esquivel-Macías et al., 2000). Para el Pensilvánico de México, se ha hallado en la Caliza Naco, Sonora (VillanuevaOlea et al., 2016), en la Formación Patlanoaya del Misuriano-Virgiliano de Puebla (EsquivelMacías et al., 2000), en el Atokano de Cerros El Tule, Sonora (Buitrón-Sánchez et al., 2012), en el Pensilvánico Medio de Sierra Agua Verde,
Sonora (Buitrón-Sánchez et al., 2007b, 2015) y, en Estados Unidos, en las formaciones Thrifty y Graham del Virgiliano de Texas (Moore y Jeffords, 1968). También hay reportes para el Pérmico de México, en la Formación Guacamaya del Leonardiano inferior de Hidalgo (EsquivelMacías et al., 2000), en la Formación Tuzancoa del Wolfcampiano-Leonardiano de Hidalgo (Arellano-Gil et al., 1998), en la Formación Olinalá del Pérmico medio de Guerrero (González-Arreola et al., 1994) y en el Pérmico medio de Cerro Los Monos en Caborca, Sonora (Buitrón-Sánchez et al., 2007a). 
Material descrito. FCMP 1345, MPLL 30, MPLL 31 .

Preptopremnum laeve Moore y Jeffords, 1968

Figuras 3.3-3.6.

Descripción. Placa circular, ligeramente achatada por deformación; faceta articular que ocupa todo el diámetro de la placa; crenulario circular y delgado con al menos 26 crénulas muy gruesas y sin bifurcaciones; areola muy ancha, plana y granulosa; lumen circular y pequeño.

Discusión. Los ejemplares de Las Delicias asignados a Preptopremnum laeve fueron comparados con P. rugosum del Pensilvánico Superior de Texas (Moore y Jeffords, 1968), pero en este último el crenulario es más grueso, con más crénulas y, en muchos casos, bifurcadas. También se comparó con Gilbertsocrinus concinnus del Osageano de la Caliza Burlington y del Misisípico de Iowa (Moore y Jeffords, 1968) que presenta una forma similar en la disposición del crenulario y la areola, pero se diferencia por una epifaceta prominente y un lumen floriforme.

Ocurrencia. Formación Las Delicias, Coahuila, México, Unidad Las Sardinas, Pérmico inferior (Kunguriano) y base de la unidad La Difunta, Pérmico medio (Wordiano-Capitaniano).

Distribución. Preptopremnum laeve se ha reportado para el Pensilvánico de Sierra Agua Verde, Sonora, México (Buitrón-Sánchez et al., 2005, 2007b, 2015), el Morrowano de Cerros El Tule, Sonora, México (Buitrón-Sánchez et al., 2012), la Lutita Mingus, Grupo Strawn del Desmoinesiano de Texas, Estados Unidos. (Moore y Jeffords, 1968), el Pensilvánico Medio-Pérmico inferior de Sierra Las Pintas, Baja California, México (Navas-Parejo et al., 2018) y el Pérmico inferior de Chiapas, México (TorresMartínez et al., 2020).

Material descrito. MPLL 32, MPLL 33, MPLL 34.

\section{Preptopremnum sp.}

Figura 3.7.

Descripción. Placa de contorno circular con perfil redondeado en vista lateral que desarrolla una epifaceta; crenulario delgado con crénulas rectas; cúlmenes finos pero más anchos que las crenelas; areola muy ancha y ligeramente granulosa; perilumen no visible; lumen de tamaño mediano y aparentemente circular.

Discusión. El ejemplar presenta un buen estado de conservación, excepto en el área correspondiente al lumen, donde no se puede saber con certeza si existe un perilumen ni la forma exacta del contorno del canal axial. Se comparó con Preptopremnum laeve del Pensilvánico Medio de Texas (Moore y Jeffords, 1968), el cual posee cúlmenes más gruesos y en menor número en comparación con el ejemplar de Coahuila. Preptopremnum rugosum, del Pensilvánico Superior de Texas (Moore y Jeffords, 1968), se puede distinguir por presentar un crenulario más ancho.

Ocurrencia. Formación Las Delicias, Coahuila, México, base de la Unidad La Difunta, Pérmico medio (Wordiano-Capitaniano).

Distribución. El género Preptopremnum se ha reportado en Estados Unidos para el Pensilvánico Medio-Superior de Texas (Moore y Jeffords, 1968). En México se le ha encontrado en el Misisípico y Pensilvánico Superior de San Salvador Patlanoaya, Puebla (Esquivel-Macías et al., 2000), en el Pensilvánico Inferior (Atokano) de Cerros El Tule, Sonora (Buitrón-Sánchez et al., 2012), en el Pensilvánico Medio de Sierra Agua Verde, Sonora (Buitrón-Sánchez et al., 2007b, 2015), en el Pensilvánico Medio-Pérmico inferior de Sierra Las Pintas, Baja California (Navas-Parejo et al., 2018), en el Pérmico inferior (Wolfcampiano-Leonardiano) de la Formación Tuzancoa (Arellano-Gil et al., 1998), en el Pérmico medio de Olinalá, Guerrero (González-Arreola et al., 1994), en el Pérmico medio de Cerros Los Monos, Caborca, Sonora (Buitrón-Sánchez et al., 2007a) y en el Pérmico inferior de Chiapas (Torres-Martínez et al., 2020).

Material descrito. FCMP 1344.

Género Heterostelechus Moore y Jeffords, 1968 Heterostelechus keithi Miller, 1968

Figura 3.8 
Descripción. Lado de la placa de muy convexo a quillado; faceta articular circular; crenulario moderadamente grande con la parte más externa elevada y un declive paulatino formando una concavidad que abarca a la areola que es granulosa; crénulas gruesas, la mayoría rectas, con crenelas más gruesas que los cúlmenes; lumen de tamaño medio y circular situado en la parte más deprimida de la faceta.

Discusión. El ejemplar presenta semejanzas morfológicas con las otras dos especies del género Heterostelechus, pero $H$. texanus del Pensilvánico Superior-Pérmico Inferior de Texas (Moore y Jeffords, 1968) y $H$. jeffordsi del Pensilvánico Superior de Texas (Moore y Jeffords, 1968) presentan una areola lisa, con un borde elevado y un hundimiento hasta al interior del lumen.

Ocurrencia. Formación Las Delicias, Coahuila, México, base de la Unidad La Difunta, Pérmico medio (Wordiano-Capitaniano).

Distribución. Se ha reportado la presencia en México de Heterostelechus keithi en la Formación Del Monte del Pensilvánico de Calnali, Hidalgo, (Buitrón-Sánchez et al., 1987), en la Formación Patlanoaya del Pensilvánico de San Salvador Patlanoaya, Puebla (Velasco y Buitrón-Sánchez, 1992), en el Pensilvánico de Chiapas (BuitrónSánchez y Solís-Marín, 1993), en la Formación La Joya del Pensilvánico de Sierra Agua Verde, Sonora (Buitrón-Sánchez et al., 2007b; Buitrón-Sánchez et al., 2015), en el Desmoinesiano-Misuriano de Cerros El Tule, Sonora (Buitrón-Sánchez et al., 2008), así como en la Formación Grupera del Pérmico inferior (Asseliano-Sakmariano) de Chiapas, México (Torres-Martínez et al., 2020). En Estados Unidos se ha reportado en la Formación Graham, Grupo Cisco del Virgiliano de Texas, (Moore y Jeffords, 1968).

Material descrito. FCMP 1346, MPLL 35, MPLL 36.

Cyclocaudiculus regularis Moore y Jeffords, 1968

Figuras 3.9, 3.10

Descripción. Columna con perfil longitudinal liso y recto; placas con al menos tres alturas diferentes; placa de contorno circular; crenulario delgado, con crénulas gruesas y rectas; areola ancha y lisa rodeando a un crenulario circular y pequeño.

Discusión. La columna presenta alteración por compactación, pero se puede apreciar que las tres placas columnares conservadas son de diferentes alturas, lo que indica que se trata de una columna heteromórfica. El ejemplar se comparó con las diferentes especies del género Preptopremnum que se distinguen por una areola granulosa. Los ejemplares Cyclocaudiculus regularis de Coahuila se compararon con especies del género Heterostelechus que se diferencian por un perfil redondeado de la columna.

Ocurrencia. Formación Las Delicias, Coahuila, México, base de la Unidad La Difunta, Pérmico medio (Wordiano-Capitaniano).

Distribución. Cyclocaudiculus regularis se ha reportado para la Formación Thrifty del Virgiliano de Texas, Estados Unidos (Moore y Jeffords, 1968). En México solo se ha reportado en la Caliza Naco del Pensilvánico de Sonora, México (VillanuevaOlea et al., 2016), y en la Formación Grupera del Asseliano-Sakmariano de Chiapas, México (Torres-Martínez et al., 2020).

Material descrito. FGMP 1347, FGMP 1348.

$$
\begin{gathered}
\text { Familia Incierta } \\
\text { Género Epicrinus gen. nov. }
\end{gathered}
$$

Diagnosis. Placa de contorno redondeado; epifaceta ancha y lisa; faceta articular mucho más pequeña que el diámetro de la placa por la presencia de epifaceta; crenulario mediano compuesto de crénulas muy gruesas; areola de tamaño pequeño; perilumen ausente; lumen circular y pequeño.

Discusión. El género nuevo que se propone no pudo ser incluido en ninguna de las familias existentes en la parataxonomía de Moore y Jeffords (1968), debido a que las características diagnósticas de la familia, como el tipo de columna y el perfil 
longitudinal de éste, no están conservadas en los ejemplares de Coahuila. Ninguno de los géneros reportados para Norteamérica presenta similitud con este material.

Etimología. El nombre del género hace referencia a la presencia de una epifaceta conspicua.

\section{Epicrinus torreonense n. sp.}

Figuras 3.11, 3.12.

Diagnosis. Ver la diagnosis del género.

Discusión. En uno de los ejemplares, la mitad del crenulario está cubierta por una capa muy delgada de sedimento que, no obstante, permite notar en la mitad interior del crenulario 24 crénulas y la areola. El ejemplar de Coahuila se diferencia de Marettocrinus angustannulus del Eifeliano-Givetiano de Polonia (Gluchowski, 1993) que presenta un perilumen elevado alrededor del lumen. Epicrinus torreonense n. sp. se distingue de Glyphidocrinus sp., del Givetiano de Polonia (Gluchowski, 1993), que se caracteriza por un crenulario más ancho y un lumen en proporción de mayores dimensiones.

Etimología. El nombre de la especie hace referencia al gentilicio de los habitantes de la ciudad de Torreón, Coahuila.

Ocurrencia. Formación Las Delicias, Coahuila, México, Unidad Las Sardinas del Pérmico inferior (Kunguriano) y base de la Unidad La Difunta del Pérmico medio (Wordiano-Capitaniano).

Ejemplares tipo. Holotipo: MPLL 37. Paratipo: MPLL 38.

\section{Discusión}

\subsection{PALEOBIOGEOGRAFÍA}

En trabajos previos se ha señalado que las faunas del Pérmico de Coahuila presentan una alta similitud con las de localidades de Texas y Nuevo México, en Estados Unidos, enmarcando esta idea en la existencia de un mar epicontinental que cubrió el centro-sur de lo que actualmente es Estados Unidos y se extendió hasta los territorios que actualmente conforman el sur de México (Guerrero-Sánchez y Quiroz-Barroso, 2013; SourTovar y Quiroz-Barroso, 2015; Torres-Martínez et al., 2016, 2019). En ese mar coexistieron múltiples taxa de fusulínidos, cefalópodos y braquiópodos, formando parte de diversas comunidades marinas de ambientes tropicales o subtropicales que caracterizan a la Provincia Grandiana (Yancey, 1975; Shen y Shi, 2004; Shen et al., 2013). En este contexto, los morfotipos de placas columnares presentes en los estratos pérmicos de la Formación Las Delicias representan el registro fósil de crinoideos, uno de los componentes más abundantes de las comunidades marinas.

En la tabla 1 se enlista a los parataxones que se describen en el presente estudio, se señala en cuáles localidades de México o de otros lugares han sido referidos, las edades de tales localidades y las fuentes bibliográficas. Es notable que, a excepción de Pentagonopternix coahuilensis, Floricyclus diminuta y Epicrinus torreonense, que se describen por primera vez, todos los parataxones encontrados en Las Delicias han sido reportados exclusivamente en localidades del Carbonífero o Pérmico de Norteamérica, incluyendo diversas localidades mexicanas. Dicha información permite hasta el momento caracterizarlos como componentes propios de las faunas del Paleozoico Superior de Norteamérica. Especies de Pentagonopternix y Floricyclus también se han encontrado en el Misisípico de Inglaterra (Moore y Jeffords, 1968) y Pérmico de Rusia (Kotlyar et al., 2006; Biakov et al., 2016), respectivamente, y su hallazgo en el Pérmico de Coahuila confirma el carácter cosmopolita de ambos géneros.

\subsection{BIOESTRATIGRAFÍA}

Como se mencionó en los párrafos anteriores, las placas columnares de crinoideos que se describen en este trabajo ya han sido referidas para diversas localidades del Carbonífero o Pérmico de México y todas tienen registros en localidades del Pensilvánico de Texas, Kansas o Colorado en Estados Unidos. La tabla 1 resalta 
Tabla 1. Distribución global de los géneros y especies determinados en el Pérmico de Coahuila, México.

\begin{tabular}{|c|c|c|c|}
\hline & Misisípico & Pensilvánico & Pérmico \\
\hline $\begin{array}{l}\text { Pentagonopternix } \\
\text { (Moore y Jeffords, } \\
\text { 1968) }\end{array}$ & & $\begin{array}{l}\text { Pensilvánico Medio de Sonora, México (Buitrón-Sánchez et al., 2015). } \\
\text { Virgiliano de Texas, E. U. (Moore y Jeffords, 1968). }\end{array}$ & $\begin{array}{l}\text { Asseliano-Sakmariano de Chiapas, } \\
\text { México (Torres-Martínez et al., 2020). } \\
\text { Roadiano-Wordiano de Transbaikal, } \\
\text { Rusia (Kotlyar et al., 2006). } \\
\text { Pérmico superior de Verkhoyansk, Rusia } \\
\text { (Biakov et al., 2016). }\end{array}$ \\
\hline $\begin{array}{l}\text { Cyclocaudex typicus } \\
\text { (Moore y Jeffords, } \\
\text { 1968) }\end{array}$ & $\begin{array}{l}\text { Merameciano- } \\
\text { Chesteriano de } \\
\text { Oaxaca, México } \\
\text { (Villanueva-Olea } \\
\text { et al., 2011). }\end{array}$ & $\begin{array}{l}\text { Morrowano-Desmoinesiano de Oaxaca, México (Villanueva-Olea et al., 2011). } \\
\text { Pensilvánico Superior de Puebla, México (Villaseñor-Martínez et al., 1987; } \\
\text { Esquivel-Macías, 1996). } \\
\text { Virgiliano de Texas, E. U. (Moore y Jeffords, 1968). }\end{array}$ & $\begin{array}{l}\text { Asseliano-Sakmariano de Chiapas, } \\
\text { México (Torres-Martínez et al., 2020). } \\
\text { Wordiano temprano-medio de Ihualtepec, } \\
\text { Oaxaca, México (Vachard et al., 1997). }\end{array}$ \\
\hline $\begin{array}{l}\text { Cyclocaudex } \\
\text { insaturatus (Moore } \\
\text { y Jeffords, 1968) }\end{array}$ & $\begin{array}{l}\text { Misisípico Medio } \\
\text { de Oaxaca, } \\
\text { México } \\
\text { (Villanueva-Olea } \\
\text { et al., 2011). }\end{array}$ & $\begin{array}{l}\text { Pensilvánico de Hidalgo, México (Buitrón-Sánchez et al., 1987). } \\
\text { Pensilvánico Inferior-Medio de Oaxaca, México (Buitrón-Sánchez et al., 2000). } \\
\text { Desmoinesiano de Kansas, E. U. (Moore y Jeffords, 1968). }\end{array}$ & $\begin{array}{l}\text { Asseliano-Sakmariano de Chiapas, } \\
\text { México (Torres-Martínez et al., 2020). }\end{array}$ \\
\hline $\begin{array}{l}\text { Floricyclus (Moore } \\
\text { y Jeffords, 1968) }\end{array}$ & $\begin{array}{l}\text { Misisípico de } \\
\text { Oaxaca, México } \\
\text { (Villanueva-Olea } \\
\text { et al., 2011). } \\
\text { Tournaisiano de } \\
\text { Devon, Inglaterra } \\
\text { (Gluchowski y } \\
\text { Racki, 2005). } \\
\text { Osageano de } \\
\text { Kentucky, E. U. } \\
\text { (Moore y Jeffords, } \\
\text { 1968). }\end{array}$ & $\begin{array}{l}\text { Pensilvánico Medio-Superior de Puebla, México (Esquivel-Macías et al., 2000). } \\
\text { Desmoinesiano de Texas, E. U. (Moore y Jeffords, 1968). } \\
\text { Desmoinesiano de Colorado, E. U. (Moore y Jeffords, 1968). } \\
\text { Desmoinesiano de Kansas, E. U. (Moore y Jeffords, 1968). } \\
\text { Virgiliano de Texas, E. U. (Moore y Jeffords, 1968). }\end{array}$ & \\
\hline $\begin{array}{l}\text { Preptopremnum } \\
\text { rugosum (Moore y } \\
\text { Jeffords, 1968) }\end{array}$ & $\begin{array}{l}\text { Misisípico de } \\
\text { Puebla, México } \\
\text { (Esquivel-Macías } \\
\text { et al., 2000). }\end{array}$ & $\begin{array}{l}\text { Pensilvánico de Sonora, México (Villanueva-Olea et al., 2016). } \\
\text { Missouriano-Virgiliano de Puebla, México (Esquivel-Macías et al., 2000). } \\
\text { Atokano de Sonora, México (Buitrón-Sánchez et al., 2012). } \\
\text { Pensilvánico Medio de Sonora, México (Buitrón-Sánchez et al., 2007b, 2015). } \\
\text { Virgiliano de Texas, E. U. (Moore y Jeffords, 1968). }\end{array}$ & $\begin{array}{l}\text { Leonardiano inferior de Hidalgo, México } \\
\text { (Esquivel-Macías et al., 2000). } \\
\text { Wolfcampiano-Leonardiano de Hidalgo, } \\
\text { México (Arellano-Gil et al., 1998). } \\
\text { Pérmico medio de Guerrero, México } \\
\text { (González-Arreola et al., 1994). } \\
\text { Pérmico medio de Sonora, México } \\
\text { (Buitrón-Sánchez et al., 2007a). }\end{array}$ \\
\hline $\begin{array}{l}\text { Preptopremnum laeve } \\
\text { (Moore y Jeffords, } \\
\text { 1968) }\end{array}$ & & $\begin{array}{l}\text { Pensilvánico de Sonora, México (Buitrón-Sánchez et al., 2005, 2007b, 2015). } \\
\text { Morrowano de Sonora, México (Buitrón-Sánchez et al., 2012). } \\
\text { Pensilvánico Medio-Pérmico inferior de Baja California, México (Navas-Parejo et } \\
\text { al., 2018). } \\
\text { Desmoinesiano de Texas, E. U. (Moore y Jeffords, 1968). }\end{array}$ & $\begin{array}{l}\text { Asseliano-Sakmariano de Chiapas, } \\
\text { México (Torres-Martínez et al., 2020). } \\
\text { Pensilvánico Medio-Pérmico inferior de } \\
\text { Baja California, México (Navas-Parejo et } \\
\text { al., 2018). }\end{array}$ \\
\hline $\begin{array}{l}\text { Heterostelechus keithi } \\
\text { (Miller, 1968) }\end{array}$ & & $\begin{array}{l}\text { Pensilvánico de Hidalgo, México (Buitrón-Sánchez et al., 1987). } \\
\text { Pensilvánico de Puebla, México (Velasco de León y Buitrón-Sánchez, 1992). } \\
\text { Pensilvánico de Chiapas, México (Buitrón-Sánchez y Solís-Marín, 1993). } \\
\text { Pensilvánico de Sonora, México (Buitrón-Sánchez et al., 2007b; Buitrón-Sánchez et } \\
\text { al., 2015). } \\
\text { Desmoinesiano-Missouriano de Sonora, México (Buitrón-Sánchez et al., 2008). } \\
\text { Virgiliano de Texas, E. U. (Moore y Jeffords, 1968). }\end{array}$ & $\begin{array}{l}\text { Asseliano-Sakmariano de Chiapas, } \\
\text { México (Torres-Martínez et al., 2020). }\end{array}$ \\
\hline $\begin{array}{l}\text { Cyclocaudiculus } \\
\text { regularis (Moore y } \\
\text { Jeffords, 1968) }\end{array}$ & & $\begin{array}{l}\text { Pensilvánico de Sonora, México (Villanueva-Olea et al., 2016). } \\
\text { Virgiliano de Texas, E. U. (Moore y Jeffords, 1968). }\end{array}$ & $\begin{array}{l}\text { Asseliano-Sakmariano de Chiapas, } \\
\text { México (Torres-Martínez et al., 2020). }\end{array}$ \\
\hline
\end{tabular}


que algunos de los parataxones encontrados en la Formación Las Delicias poseen una distribución temporal muy amplia y que pueden encontrarse en localidades con edades del Carbonífero y Pérmico. Un caso extremo es Cyclocaudex typicus, que se conoce para el Osageano (Misisípico inferior) de Oaxaca y tiene registros con edades pensilvánicas en Oaxaca, Puebla y Texas, e inclusive se ha reportado para el Pérmico inferiormedio de Oaxaca y Chiapas. Caso similar es el de Preptopremnum rugosum que se ha referido para el Misisípico de Puebla, Pensilvánico de Sonora y Puebla, y Pérmico inferior-medio de Hidalgo. El resto de los parataxones presentes en el Pérmico de Coahuila se han reconocido previamente en diversas localidades de edades misisípicopensilvánicas (Cyclocaudex insaturatus y el género Floricyclus) o pensilvánico-pérmicas (el género Pentagonopternix, Cyclocaudex insaturatus, Preptopremnum laeve y Cyclocaudiculus regularis). Heterostelechus keithi solo se conocía para afloramientos pensilvánicos de Puebla, Chiapas y Sonora en México, para el Virgiliano de Texas en Estados Unidos y el Cisuraliano de Chiapas en México. Fuera de Norteamérica únicamente existen los registros de Pentagonopternix que se conocen para el Pérmico superior de Verkhoyansk, Rusia y del género Floricyclus presente en el Tournaisiano de Devon, Inglaterra.

Las edades conocidas para cada uno de los parataxones de placas columnares de crinoideos presentes en Coahuila permiten señalar que carecen de un valor bioestratigráfico significativo. Este sesgo se puede relacionar con varias causas: una de ellas es considerar que la morfología de las placas columnares de crinoideos no es una variable que permita caracterizar a diferentes géneros o especies, o que tales estructuras han evolucionado con tasas muy lentas e independientes de las tasas de evolución taxonómica de los crinoideos. Si esto es real, la ausencia de registros de edad pérmica de la mayoría de los parataxones listados en este trabajo se explica por el bajo número de trabajos publicados en que se realiza la descripción de placas columnares, que es el tipo de fósiles que se encuentran en abundancia y, comúnmente, en la mayoría de las asociaciones fósiles de ambientes marinos. Para todos los parataxones presentes en Las Delicias se comprueba su existencia en el Pérmico inferior o medio.

\section{Agradecimientos}

A. D. Navarro-Santillán por su asistencia técnica, al equipo editorial del BSGM y a la Dirección General de Asuntos del Personal Académico (DGAPA), Universidad Nacional Autónoma de México, por el apoyo económico a través del proyecto PAPIIT IA102618.

\section{Referencias}

Arellano-Gil, J., Vachard, D., Yussim, S., Flores de Dios-González, A., 1998, Aspectos estratigráficos, estructurales y paleogeográficos del Pérmico Inferior al Jurásico Inferior en Pemuxco, estado de Hidalgo, México: Revista Mexicana de Ciencias Geológicas, 15, 9-13.

Biakov A. S., Zakharov Yu. D., Horacek M., Richoz S., Kutygin R. V., Ivanov Yu. Yu., Kolesov E. V., Konstantinov A. G., Tuchkova M. I., Mikhalitsyna T. I., 2016, New data on the structure and age of the terminal Permian strata in the South Verkhoyansk region (northeastern Asia): Russian Geology and Geophysics, 57, 282-293. https://doi. org/10.1016/j.rgg.2016.02.005

Buitrón-Sánchez, B.E., Solís-Marín, F.A., 1993, La biodiversidad en los equinodermos fósiles y recientes de México: Revista de la Sociedad Mexicana de Historia Natural, 44, 209-231.

Buitrón-Sánchez, B.E., Patiño, J., Moreno, A., 1987, Crinoideos del Paleozoico Tardío de Calnali, Hidalgo: Revista de la Sociedad Mexicana de Paleontología, 1, 125-136.

Buitrón-Sánchez B.E., Flores de Dios-González A., Vachard D., 2000, Nuevos hallazgos 
de invertebrados paleozoicos (ScyphozoaConulata, Archaeogastropoda-Trochina y Echinodermata-Crinoidea) en la región de Nochixtlán-Ixtaltepec, Oaxaca: Geos, 20(3), 298.

Buitrón-Sánchez B.E., Almazán-Vázquez E., Vachard D., Gómez-Espinosa C., MendozaMadera C., 2005, Crinoides pensilvánicos asociados a facies "arrecifales" de chaetetidos en Sierra Agua Verde, estado de Sonora, México: Geos, 25(1), 151.

Buitrón-Sánchez, B.E., Almazán-Vázquez, E., Vachard, D., 2007a, Middle Permian crinoids (Echinodermata, Crinoidea) from Cerros Los Monos, Caborca, Sonora, Mexico and paleogeographic considerations: Revista Mexicana de Ciencias Geológicas, 24, 344-353

Buitrón-Sánchez, B.E., Gómez-Espinosa, C., Almazán-Vázquez, E., Vachard, D., 2007b, A late Atokan regional encrinite (early late Moscovian, Middle Pennsylvanian) in the Sierra Agua Verde, Sonora State, NW Mexico, en Álvaro, J.J., Aretz, M. Boulvain, F., Munnecke, A., Vachard, V. Vennin, E. (eds.), Palaeozoic reefs and bioaccumulations: climatic and evolutionary controls (275): London, Geological Society, Special Publications, 201-209. http://dx.doi. org/10.1144/GSL.SP.2007.275.01.13

Buitrón-Sánchez, B.E., Gómez-Espinosa, C., Almazán-Vázquez, E., Vachard, D., Laguarda-Figueras, A., Solís-Marín, F., 2008, A review of the crinoid columnals (Echinodermata-Crinoidea) from the Carboniferous of Mexico: Revista de Biología Tropical, 56, 1-12.

Buitrón-Sánchez, B.E., Vachard, D., AlmazánVázquez, E., Palafox-Reyes, J.J., 2012, Una secuencia cratónica del Carbonífero al Pérmico inferior expuesta en los Cerros El Tule, noreste de Sonora, México: Revista Mexicana de Ciencias Geológicas, 29, 39-62. Buitrón-Sánchez, B.E., Chacón-Wences, O., Vachard, D., Palafox-Reyes, J.J.,
Jiménez-López, J.C., Sour-Tovar, F. 2015, Pennsylvanian biota of the Sierra Agua Verde, Sonora, Mexico: biostratigraphic and paleogeographic considerations: Revista Mexicana de Biodiversidad, 86, 521-527. http://dx.doi.org/10.1016/j. rmb.2015.04.006

Coney, P. J., Campa-Uranga, M. F., 1987, Lithotectonic terrane map of Mexico (west of the 9151 meridian): US. Geological Survey, Miscellaneous Field Studies Map MF-1874-D, scale 1:2,500,000. https://doi. org $/ 10.3133 / \mathrm{mf1} 874 \mathrm{a}$

Esquivel-Macías, C., 1996, Invertebrados del Paleozoico Tardío de las regiones de Olinalá, Guerrero y Patlanoaya, Puebla. Tesis de maestría, Universidad Nacional Autónoma de México, Facultad de Ciencias, 123 pp.

Esquivel-Macías, G., Ausich W.I., BuitrónSánchez, B.E., Flores de Dios, A., 2000, Pennsylvanian and Mississippian pluricolumnal assemblages (Class Crinoidea) from southern Mexico and a new occurrence of a column with a tetralobate lumen: Journal of Paleontology, 74, 1187$1190 . \quad$ https://doi.org/10.1666/0022$3360(2000) 074<1187$ :PAMPAC $>2.0$. $\mathrm{CO} ; 2$

Gluchowski, E., 1993, Crinoid assemblages in the Polish Givetian and Frasnian: Acta Palaeontologica Polonica, 38, 35-92.

Gluchowski, E., Racki, G., 2005, Disarticulated crinoid stems from the Devonian and Carboniferous of north Devon, England: Proceedings of the Yorkshire Geological Society, 55 (3), 161-172. http://dx.doi. org/10.1144/pygs.55.3.161

González-Arreola, C., Villaseñor-Martínez, A.B., Corona-Esquivel, R., 1994, Permian fauna of the Los Arcos Formation, municipality of Olinalá, state of Guerrero, Mexico: Revista Mexicana de Ciencias Geológicas, 11(2), 214-221.

Guerrero-Sánchez, M., Quiroz-Barroso, S.A., 2013, Bivalvos guadalupianos (Pérmico 
medio) de la Formación Olinalá, Guerrero, México: Boletín de la Sociedad Geológica Mexicana, 65(3), 533-551. https://doi. org/10.18268/bsgm2013v65n3a8

King, R.E., Dumbar, C.O., Cloud, P.E., Miller, A.K., 1944, Geology and Paleontology of the Permian area northwest of Las Delicias, southwestern Coahuila, Mexico: Geological Society of America Special Paper (52), 1-170. https://doi.org/10.1130/spe52-p1

Kotlyar G. V., Popeko, L. I., Kurilenko A. V., 2006, The Permian of the Transbaikal region, eastern Russia: Biostratigraphy, correlation and biogeography: Journal of Asian Earth Sciences, 26, 269-279. https:// doi.org/10.1016/j.jseaes.2005.08.003

McKee, J. W., Jones, N. W., Long, L. E., 1990, Stratigraphy and provenance of strata along the San Marcos fault, central Coahuila, Mexico: Geological Society of America Bulletin, 102, 593-614. https://doi. org/10.1 130/0016-7606(1990)102<0593:sa posa $>2.3 . c 0 ; 2$

McKee, J.W., Jones, N.W., Anderson, T.H., 1999, Late paleozoic and early mesozoic history of the Las Delicias terrane, Coahuila, Mexico, en Bartolini, G., Wilson, J.L., Lawton, T.F., (eds.), Mesozoic sedimentary and Tectonic history of north-central Mexico: Boulder, Colorado, Geological Society of America Special Paper (340), 161-189. https://doi. org/10.1130/0-8137-2340-x.161

Moore, R.C., Jeffords, R.M., 1968, Classification and nomenclature of fossil crinoids based on studies of dissociated parts of their columns: The University of Kansas Paleontological Contributions, Serial Number 46, Article 9, 1-86.

Navas-Parejo, P., Lara-Peña, R.A., TorresMartínez, M.A., Martini, M., 2018, Biostratigraphy and petrography of upper Paleozoic rocks of Sierra Las Pintas, northern Baja California: Journal of South American Earth Sciences, 84, 160-171. https://doi. org/10.1016/j.jsames.2018.03.010

Quiroz-Barroso, S.A., Sour-Tovar, F.,
Quiroz-Barragán, J., 2019, Dos especies nuevas de Paraconularia (Scyphozoa, Conulariidae) en la Formación Las Delicias, Pérmico inferior-medio de Coahuila, México: Revista Brasileira de Paleontología, 22(2), 120-130. https://doi:10.4072/ rbp.2019.2.04

Rosales-Lagarde, L., Centeno-García, E., Dostal J., Sour-Tovar, F. Ochoa-Camarillo, H., Quiroz-Barroso, S.A., 2005, The Tuzancoa Formation: evidence of an Early Permian Submarine Continental Arc in EastCentral Mexico: International Geology Review, 47(9), 901-919. https://doi. org/10.2747/0020-6814.47.9.901

Sedlock, R. L., Ortega-Gutiérrez, F., Speed, R. C.,1993, Tectonostratigraphic terranes and tectonic evolution of Mexico: Geological Society of America Special Paper (278), 76 77. https://doi.org/10.1130/spe278-pl

Shen, S.Z., Shi, G.R., 2004, Capitanian (Late Guadalupian, Permian) global brachiopod paleobiogeography and latitudinal diversity pattern: Palaeogeography, Palaeoclimatology, Palaeoecology, 208, 235-262. http://doi.org/10.1016/j. palaeo.2004.03.009

Shen, S.Z., Zhang, H., Shi, G.R., Li, W.Z., Xie, J.F., Mu, L., Fan, J.X., 2013, Early Permian (Cisuralian) global brachiopod palaeobiogeography: Gondwana Research, 24, 104-124. https://doi.org/10.1016/j. gr.2012.05.017

Sour-Tovar, F., Quiroz-Barroso, S. A., 2015, Lower-Middle Permian brachiopods from two localities of Central-South Mexico: faunas of a closing ocean. Permophiles: Newsletter of the Subcommission on Permian Stratigraphy, 61, 82-84.

Torres-Martínez, M.A., Sour-Tovar, F., Barragán, R., 2016, Permian(Leonardian) brachiopods from Paso Hondo Formation, Chiapas, southern Mexico. Paleobiogeographical implications: Journal of South American Earth Sciences, 71, 71-81. https://doi. org/10.1016/j.jsames.2016.06.012 
Torres-Martínez, M. A., Heredia-Jiménez, D. P., Quiroz-Barroso, S. A., Navas-Parejo, P., Sour-Tovar, F., Quiroz-Barragán, J., 2019, A Permian (late Guadalupian) brachiopod fauna from northeast Mexico and their paleobiogeographic affinities: Journal of South America Earth Sciences, 92, 41-55. https://doi.org/10.1016/j. jsames.2019.03.002

Torres-Martínez, M.A., Villanueva-Olea, R., Sour-Tovar, F., 2020, Columnar ossicles of Permian crinoids, including two new genera, from the Grupera Formation (AsselianSakmarian) of Chiapas, Mexico: Boletín de la Sociedad Geológica Mexicana,72(2), A290819. https://doi.org/10.18268/ bsgm2020v72n2a290819

Vachard, D., Flores de Dios, A., Buitrón-Sánchez, B.E., 1997, Sur une nouvelle localitéà fusulines du Wordien (Permien Supérieur) du Mexique: Conséquences paléogéographiques: Geobios, 30, 361-370. https://doi.org/10.1016/ S0016-6995(97)80196-9

Velasco de León, P., Buitrón-Sánchez, B.E., 1992, Algunos crinoideos (EchinodermataCrinoidea) del Misisípico-Pensilvánico de San Salvador Patlanoaya, estado de Puebla: Revista de la Sociedad Mexicana de Paleontología, 5, 71-81.

Villanueva-Olea, R., Castillo-Espinoza, K.M., Sour-Tovar, F., Quiroz-Barroso, S.A.,
Buitrón-Sánchez, B.E., 2011, Placas columnares de crinoides del Carbonífero de la región de Santiago Ixtaltepec, Municipio de Nochixtlán, Oaxaca: consideraciones estratigráficas y paleobiogeográficas: Boletín de la Sociedad Geológica Mexicana, 63, 443-457. http://dx.doi.org/10.18268/ BSGM2011v63n3a5

Villanueva-Olea, R., Buitrón-Sánchez, B.E., Palafox-Reyes, J.J., Piña-Flores, S., 2016, Crinoides (Echinodermata: Crinoidea) del Pensilvánico de sierra Las Mesteñas, NE de Sonora, México: Revista Mexicana de Biodiversidad, 87, 1225-1234. https://doi. org/10.1016/j.rmb.2016.10.014

Villaseñor-Martínez, A.B., Martínez, A., Contreras D.B., 1987, Bioestratigrafía del Paleozoico Superior de San Salvador Patlanoaya, Puebla, México: Revista de la Sociedad Mexicana de Paleontología, 1, 396-417.

Wardlaw, B.R., Furnish, W.M., Nestell, M.K., 1979, Geology and Paleontology of the Permian beds near Las Delicias, Coahuila, México: Geological Society of America Bulletin 90,111-116. https://doi. org/10.1130/0016-7606(1979)90\%3C111:g apotp $\% 3 \mathrm{E} 2.0$. co;2

Yancey, T.E., 1975, Permian marine biotic provinces in North America: Journal of Paleontology, 49, 758-766. 